\title{
Importance of Oxidative Stress Marker in Healthy Females Undergoing Assisted Reproductive Technology
}

Parijatham S*, Saikumar P and Chandra Selvi E

Sree Balaji Medical College and Hospitals, Bharath University, Chennai, Tamil Nadu, India

\begin{abstract}
Background: Females with primary infertility are subjected to various investigations. Most of them are subjected for stimulation of ovulation. Before subjecting them for stimulation, case selection is important. Knowledge about the basic problems involved in infertility is very important. The male factor should be ruled out. Whatever the reason may be, the females bare the sole responsibility. In infertile couples, oxidative stress (OS) in the females involved where male factor is the cause is estimated. Because the mental stress they face in the society may increase the levels of oxidative stress in them.
\end{abstract}

Aim: Serum level of oxidative stress marker Malondialdehyde (MDA) was done in females in infertile couples where the male factor plays the causative factor. Estimated levels were compared with the MDA levels of fertile females.

Materials and methods: 17 infertile couples who were attending fertility clinic at Sree Balaji Medical College and Hospital were selected. In them, the male factor was the reason for infertility. These 17 females were included in the study where the female factors were normal and age, height and weight matched 25 females were included as controls.

Results: MDA levels were high $(2.8 \pm 0.08)$ in females where other fertility factors were normal compared with fertile females $(0.91 \pm 0.13)$

Conclusion: In apparently healthy females where male factor is the etiology, failure to conceive by Assisted Reproductive Technology (ART) may be due to much reason. Increased levels of MDA may be one of the reasons. This may produce difficulty in conception by Assisted Reproductive Technology (ART).

Keywords: Infertility; Oxidative stress; MDA; Assisted reproductive technology

\section{Introduction}

WHO (1991) defines infertility as "failure to conceive despite two years of cohabitation exposure to pregnancy. If the couple has never conceived despite cohabitation and exposure to pregnancy (not contracepting) for a period of two years, it is called primary infertility" [1]. Infertility has been defined differently. Infertility is the inability to conceive after one year of regular intercourse without contraceptive use is the main medical definition.

Prevalence rates of infertility estimation are not accurate. A lot of research had been done about high fertility and regularising the growing population. On the other hand infertility has become one of the biggest problems nowadays. Country like India, where the population is high also face this problem. In countries with high fertility rates, infertility tends to be highest, an occurrence termed as 'barrenness amid plenty' [2]. Age, weight, smoking, alcohol, caffeine use, exposure to environmental hazards (pesticide, industrial solvents, phthalates, etc.) and stress are the main risk factors. Women who fail to conceive have high stress levels. It is found to be equivalent to that of women with cancer. In this silent problem, even though men and women are affected equally, in developing countries, women bear the blame more than men. Unable or difficult to conceive is a physically and psychologically painful condition in a female's life. Unfortunately women were blamed and they do things because of ignorance or lack of counselling. In cases where male partner has the problem the society still blames female partner. She goes through unwanted investigations, repeated stimulation for ovulation and takes lots of medications which lead to unwanted side effects.

Some agents modulate the physiological process of female reproductive system and are called free radicals. The reactive oxygen species and oxygen radicals may produce some damages to the reproductive tract. So estimation of OS marker (MDA) level is necessary in all the females who attend the infertility clinic.

\section{Materials and Methods}

Inclusion Criteria: The selected female volunteers were aged between 19 to 35 years. 17 females were selected who never conceived even one year of married life aged between 19 to 40 years. The female subjects included had normal physiological and other factors for conception, but their male partner's Semen analysis showed abnormal results.

The infertility was due to male factor. The female factor like PID, Tubal factor, PCOS and hormonal factors were normal in these females. Their husband's semen analyses were abnormal. 25 controls were included in the study. The control group was selected belonging to the same age, height and weight. In control group minimum one child birth was considered as fertility.

After getting an explained, written, informed consent from the subjects, height, and weight of the subjects were measured to calculate the body mass index.

*Corresponding author: Parijatham S, Sree Balaji Medical College and Hospitals, Bharath University, Chennai, Tamil Nadu, India, Tel: +9144 22276950; E-mail: dr_parijatham@yahoo.com

Received August 19, 2015; Accepted October 31, 2015; Published November 03, 2015

Citation: Parijatham S, Saikumar P, Chandra Selvi E (2015) Importance of Oxidative Stress Marker in Healthy Females Undergoing Assisted Reproductive Technology. J Environ Anal Toxicol 5: 331. doi:10.4172/2161-0525.1000331

Copyright: @ 2015 Parijatham S, et al. This is an open-access article distributed under the terms of the Creative Commons Attribution License, which permits unrestricted use, distribution, and reproduction in any medium, provided the original author and source are credited. 
Routine investigations were done to rule out other co- morbid conditions.

1. Hemoglobin, Total Count, Differential Count, Erythrocyte Sedimentation Rate, Random Blood Sugar, Blood Grouping and typing, HIV, HbsAg

\section{Mantoux}

3. Urine Examination: -sugar, albumin, microscopy examination.

All the hormone levels (thyroid profile, oestrogen, progesterone, $\mathrm{LH}$ ), tubal factor were assessed prior to the study.

Semen analysis of the male counterpart was done prior to the study.

Venous blood samples were collected from the subjects in the empty tubes. Collected blood samples were subjected to the estimation of MDA. Serum Malondialdehyde (MDA) as a marker was estimated. TBARS (Thiobarbituric Acid Reactive Substances) method was used to estimate serum MDA. All the investigations were done at the Central Laboratory and in the Department of Physiology of Sree Balaji Medical College and Hospital. All values were presented as mean \pm standard deviation. Statistical analysis was done by ' $\mathrm{t}$ ' test and ANOVA method using SPSS software. MDA levels were compared between cases and control (Table 1).

In infertile couples, where male factor was the reason, the female partner's MDA which was shown as cases was increased (2.80 \pm 0.08$)$ compared to control group and it was statistically significant.

\section{Discussion}

The birth of Louise Brown in 1978 changed the scenario in the treatment of infertility. Patrick Steptoe and Robert Edwards introduced the technique of In vitro Fertilization (IVF) to the world. Edwards won the 2010 Nobel Prize for this work. The world's second and India's first IVF baby, Kanupriya, alias Durga, was born on October 3, 1978. All the infertile couples cannot afford such time consuming and costly procedure. Still the search to solve the problem is going on.

There are many studies focussing on hormones in relation with infertility. Studies on the effect of oxidative stress due to free radicals are comparatively less. Oxygen's by products are free radicals. Unstable oxygen molecule can damage DNA and cell and these damages are prevented by anti-oxidants. These anti-oxidants bind to this unstable molecule stopping the damage. Normally balance between antioxidants and free radical exists. More free radical production or low antioxidants level causes oxidative stress.

Mitochondrial mutations in different complexes of electron transport chain have been reported to disrupt the electron flow which leads to formation of more superoxide ions or more Reactive Oxygen Species (ROS) [3]. Study done in mouse by Chao et al. [4] showed more aggregated mitochondria in the cytoplasm of the repetitively stimulated embryos. Repeated stimulation of the ovary in more cycles results in oxidative damage including $8-\mathrm{OH}-\mathrm{dG}$, lipoperoxide, and carbonyl proteins. More mitochondrial DNA (mtDNA) mutations were also seen. Increased carbonyl proteins were also seen after repeated stimulation. Zelter et al. suggested chronic stimulation of

\begin{tabular}{|c|c|c|}
\hline Parameters & Cases $(\mathbf{n}=\mathbf{1 7})$ & Control $(\mathbf{n}=\mathbf{2 5})$ \\
\hline MDA $(\mathrm{nmol} / \mathrm{ml})$ & $2.8 \pm 0.08^{* *}$ & $0.91 \pm 0.13$ \\
\hline
\end{tabular}

${ }^{* *} p<0.05$ statistically significant when compared to controls

Table 1: Oxidative Stress Marker (MDA) in Serum of Women among Infertility couple where the aetiology was the male factor (Mean $\pm \mathrm{SD}$ ). peritoneal fluid macrophages induce the release of ROS. Iborra et al. [5] states that chronic inflammatory response and the presence of a local oxidative environment could play an important role in reproductive system. An altered protein function and the presence of circulating auto antibodies to new epitopes, such as malondialdehyde bound to proteins, block some membrane surface antigens with a receptor function in the reproductive system. Sperm capacitation, oocyte fertilization, or embryo implantation may be inhibited due to oxidative stress and chronic inflammatory conditions. Serum MDA in women with infertility due to male partner was increased in our study. It may be due to the other factors that are contributing to oxidative damage. The chances of conception are decreased in these subjects. It may be due to psychological [6], drug induced oxidative damage as a result of repeated ovarian stimulation [5].

In cases, where male factor is the reason for failure to conceive various ART are tried. Females undergo lots of procedures and on various medications. This may result in increased oxidative stress in them.

\section{Conclusion}

The increased level of oxidant is seen in women group where the male factor is the main cause. The MDA levels were increased in women of the study population compared with the fertile women. Even though the study group females did not have any problem for normal conception the MDA results were high. The mechanism mentioned earlier may be the reason for the increased level of MDA level in females compared with the fertile female's level of MDA where the male factor is the reason for failure to conceive. This study has to be followed for further evaluation. Apparently normal women during routine infertility workup, where the male factor is involved may fail to conceive in ART, if other factors causing oxidative stress and antioxidant status are not ruled out [7]. Hence the estimation of oxidant and total antioxidant status is a useful indicator explaining the imbalance between the oxidant and total antioxidant status leading to many problems in females who are undergoing ART. ART is a boon in the treatment of infertility. By correcting the total antioxidant status in infertile couple by proper food intake and supplementation with antioxidants there may be a fruitful result. Especially in women with unexplained aetiology and also in ART this antioxidant treatment might help.

\section{References}

1. World Health Organization (2000) Reproductive health indicators for global monitoring: Report of the second interagency meeting. WHO, Geneva. p: 23.

2. Van Balen F, Gerrits T (2001) Quality of infertility care in poor-resource areas and the introduction of new reproductive technologies. Hum Reprod 16: $215-$ 219.

3. Kumar M, Pathak D, Kriplani A, Ammini AC, Talwar P, et al. (2010) Nucleotide variations in mitochondrial DNA and supra-physiological ROS levels in cytogenetically normal cases of premature ovarian insufficiency. Arch Gynecol Obstet 282: 695-705.

4. Chao HT, Lee SY, Lee HM, Liao TL, Wei YH, et al. (2005) Repeated ovarian stimulations induce oxidative damage and mitochondrial DNA mutations in mouse ovaries. Ann N Y Acad Sci 1042: 148-156.

5. Iborra A, Palacio JR, Martinez $P$ (2005) Oxidative stress and autoimmune response in the infertile woman. Chem Immunol Allergy 88: 150-162.

6. Tsuboi H, Tatsumi A, Yamamoto K, Kobayashi F, Shimoi K, et al. (2006) Possible connections among job stress, depressive symptoms, lipid modulation and antioxidants. J Affect Disord 91: 63-70.

7. Veena BS, Sharmila U, Satish KA, Pratap KN (2008) Evaluation of Oxidative Stress, Antioxidants and Prolactin in Infertile Women. Indian J Clin Biochem 23: $186-190$. 